\title{
Real-time Fusion of ECG and SpO2 Signals to Reduce False Alarms
}

\author{
Jianwei Su, Sanchao Liu, Zehui Sun, Bailei Sun, Wenyu Ye, Cadathur Rajagopalan*, Xianliang He \\ Mindray, Shenzhen, China
}

\begin{abstract}
The aim of this study was the reduction of false arrhythmia alarms and improvement in the accuracy of calculated heart rates $(H R)$ in real-time patient monitors by the fusion of information from ECG and $\mathrm{SpO} 2$ signals. These signals were analyzed independently to derive features for use in the fusion analysis. Information regarding the detection and classification of QRSs, signal quality index (SQI), HR and arrhythmia alarms were obtained from the ECG. Pulses, signal quality, pulse rate $(P R)$ and hemodynamic parameters were obtained from the SpO2. Independent results from each signal were confirmed by fusing features from both. When an arrhythmia alarm was triggered, corresponding $\mathrm{SpO} 2$ features were checked to determine whether the alarm should be accepted or rejected. $H R$ and PR reliability was estimated using signal quality while QRSs and SpO2 pulses were matched to exclude spurious beats due to motion or other artifacts. Thus, calculated $H R$ and PR were more accurate and false HRs from noisy ECG signals could be supplanted by the pulse rate if noise free. Adult, pediatric and neonate ECG signals were collected to make up training and test datasets. False alarm suppression was over $50 \%$ for all arrhythmia calls while it was over $60 \%$ for life threatening alarms. False HR/PR was reduced by $80 \%$.
\end{abstract}

\section{Introduction}

Research has shown that a high percentage of clinical alarms are either false or non-actionable. Alarm fatigue related to such false alarms has been on ECRI's list of Top 10 Health Technology Hazards [1]. High rates of false alarms are associated with negative consequences like delayed response times, missed true events, decline in the quality of patient care, increased length of patient stay and increased hospital costs.

The reduction of false alarms has been a key issue in patient monitoring over the past decade. One method to address this issue is based on processing only one type of signal including signal quality assessment to determine whether to trigger the alarm or not [2]. Another is the use of multi-lead ECG analysis to improve the detection and classification of QRS complexes by selecting leads without artifacts [3]. Yet another technique to suppress false alarms is based on integrating information from correlated signals, such as ECG, arterial blood pressure (ABP) and photoplethysmogram (PPG). Algorithms using $\mathrm{ABP}$ and PPG waveform features were developed to reduce the incidence of false critical ECG arrhythmia alarms [4-7].

In this article, a method for the real-time fusion of ECG and $\mathrm{SpO} 2$ signals to reduce false arrhythmia alarms and improve the accuracy of calculated HR/PR is discussed. In addition to pulse waveform features, hemodynamic parameters, such as oxygen saturation and perfusion index (PI), obtained from the $\mathrm{SpO} 2$ signal were used to suppress false arrhythmia alarms and avoid rejecting true critical arrhythmia events.

\section{Method}

The proposed fusion analysis algorithm includes two main steps (Figure 1). First, the ECG and $\mathrm{SpO} 2$ signals are analyzed separately to derive independent features and parameters (HR/PR, ARR alarms...) used as input for the data fusion analysis. Independent results from each signal are confirmed by data fusion analysis where the first step is to learn noise-free ECG and $\mathrm{SpO} 2$ signals so that conditions not appropriate for data fusion can be determined. If the ECG and $\mathrm{SpO} 2$ signals are suitable for fusion then HR/PR confirmation as well as ARR alarm validation are carried out.

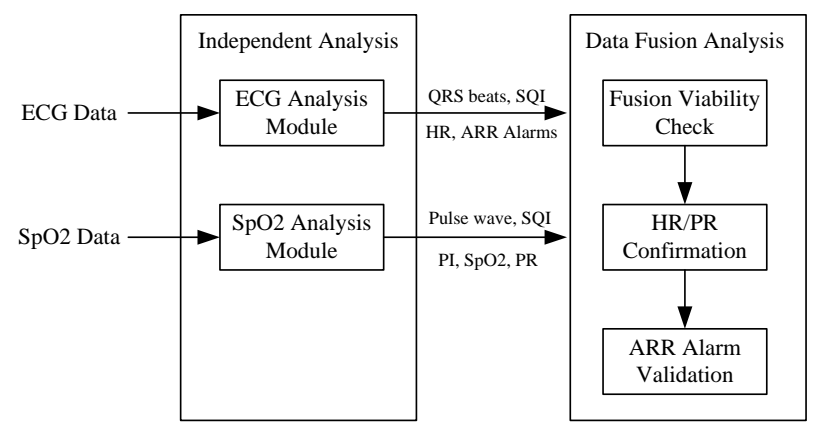

Figure 1. Schematic diagram of the real-time fusion of ECG and SpO2 Signals. 


\subsection{ECG Analysis Module}

The ECG analysis module is a real-time multi-channel ECG processing system. QRS complexes from each lead are detected and classified first. Then a QRS matching scheme is used to link them as a group of matched QRS complexes based on synthesizing the information from each lead. HR values and ARR alarms are generated based on the synthesis of the QRSs. Meanwhile, a SQI varying from 0 to 4 (level 0 represents a noise free signal while level 4 indicates the worst signal quality) is calculated each second to determine the noise level in the ECG signal [2].

\subsection{SpO2 Analysis Module}

This $\mathrm{SpO} 2$ signal is analysed via time domain and frequency domain techniques in this module to obtain oxygen saturation values and PR [8]. Pulse interval, amplitude, onset and offset are computed in real-time for each pulse. The SQI level (good or bad) associated with each pulse is generated based on similarity between the current pulse and recent pulses. The pulse interval is compared to recent pulses with good SQI to determine whether the pulse rhythm is regular. In addition, PI, an indicator of $\mathrm{SpO} 2$ signal strength, is used to exclude low perfusion signals that might not be suitable for critical arrhythmia alarm validation. Low perfusion signals are a lot more susceptible to artifacts and features derived from them might be unreliable.

\subsection{Fusion Viability Check}

Noise free ECG and SpO2 signals are continuously compared to ensure they are suitable for data fusion when needed. Generally, changes in electrical activity (ECG signal) of the heart cause changes in cardiovascular circulation ( $\mathrm{SpO} 2$ signal). However, in the presence of blockages or peripheral vascular disease, ECG and $\mathrm{SpO} 2$ signals may not be in sync thus rendering them inappropriate for fusion. When the $\mathrm{SpO} 2$ value is very low (below 85\%), even if the pulses are clean and periodic, fusion is not carried out. In this module QRS complexes and $\mathrm{SpO} 2$ pulses within a window are compared to verify synchronization between the two signals and fusion analysis is carried out only when the same rhythm is shown to be manifest in both signals. Otherwise, the HR/PR and ARR alarms will remain unchanged.

\subsection{HR/PR Confirmation}

This process is triggered when the difference between HR and PR exceeds a predefined threshold. The reliabilities of $\mathrm{HR}$ and PR are estimated using SQIs of the corresponding QRS complexes and $\mathrm{SpO} 2$ pulses within a confirmation window which is the same as that used to compute average HR/PR values. If half of the QRS beats or pulses within the window are deemed to have poor signal quality, HR or PR will be considered unreliable. Beats from the reliable and unreliable signals are matched to exclude spurious or artificial beats. The HR calculated using matched beats from the reliable signal is compared to the historical trend of HR and PR to confirm it is reliable. If confirmed, the reliable HR/PR will supplant the false PR/HR. An example of false HR value from the ECG being replaced by the PR is illustrated in Figure 2.

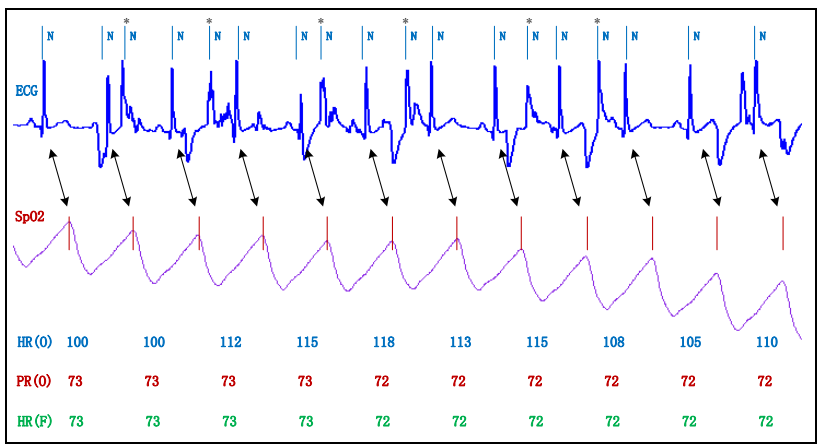

Figure 2. An example of HR confirmation using clean $\mathrm{SpO} 2$ signal. HR values were affected by spurious beats due to electrode artifact and not easily excluded using only the ECG signal. PR values were reliable since the $\mathrm{SpO} 2$ signal quality was good and the pulse rhythm was regular. Using the pulses identified on the $\mathrm{SpO} 2$ signal, QRSs (indicated by asterisks), were considered unreliable and excluded from HR calculations. As a result, the erroneous HR values were replaced by the corresponding $\mathrm{PR}$ values.

\subsection{ARR Alarm Validation}

When an ARR alarm is triggered by the ECG analysis module, corresponding $\mathrm{SpO} 2$ features within a validation window are checked to determine whether the alarm should be accepted or rejected. For life threatening alarms, the validation window is mainly determined by the starting point of the event and the time at which the alarm is generated. For other alarms, the window is determined by the definition used for the alarm condition. The basic validation rules used for arrhythmia alarms are:

(1) Asystole (ASY)

If the median $\mathrm{SpO} 2$ value is greater than $90 \%$, the median PI value is above $1.0,80 \%$ of the pulses indicate good quality and the pulse rhythm is regular within the validation window, the ASY alarm is rejected; otherwise, the alarm is retained. An example of false ASY alarm rejection is shown in Figure 3.

(2) Ventricular fibrillation (VFIB)

If abnormal pulses (large changes in pulse interval or ratio of valley to peak time and peak to valley time of a 
pulse) are not present, the median $\mathrm{SpO} 2$ value is greater than $90 \%$, the median PI value is above $1.0,80 \%$ of the pulses indicate good quality, and the pulse rhythm is regular within the validation window, the VFIB alarm is rejected; otherwise, the alarm is retained.

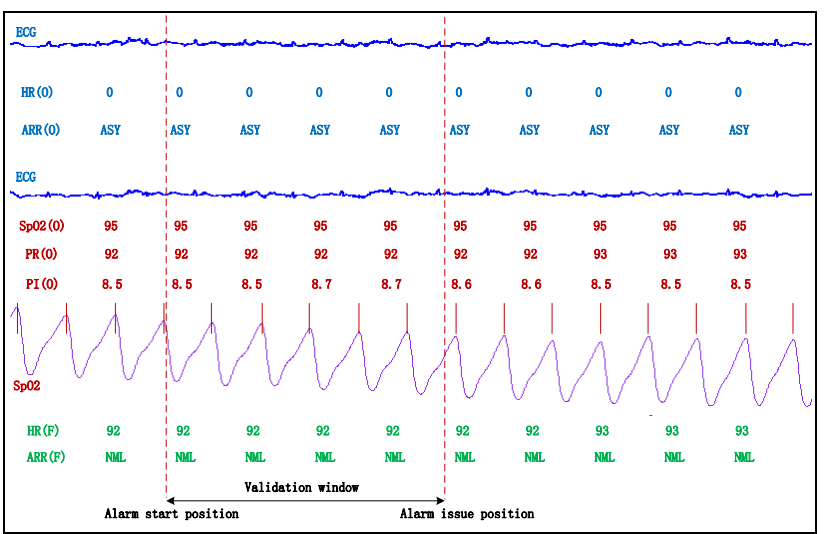

Figure 3. An example of false ASY alarm suppression. Low amplitudes of signals on both ECG leads resulted in QRSs not being detected and an ASY alarm being called. The corresponding $\mathrm{SpO} 2$ signal indicates the presence of robust beats. For each second within the validation window determined by the ASY threshold, $\mathrm{SpO} 2$ features were checked following the pre-defined rules. The false ASY alarm was rejected and the HR value was replaced by the corresponding PR value.

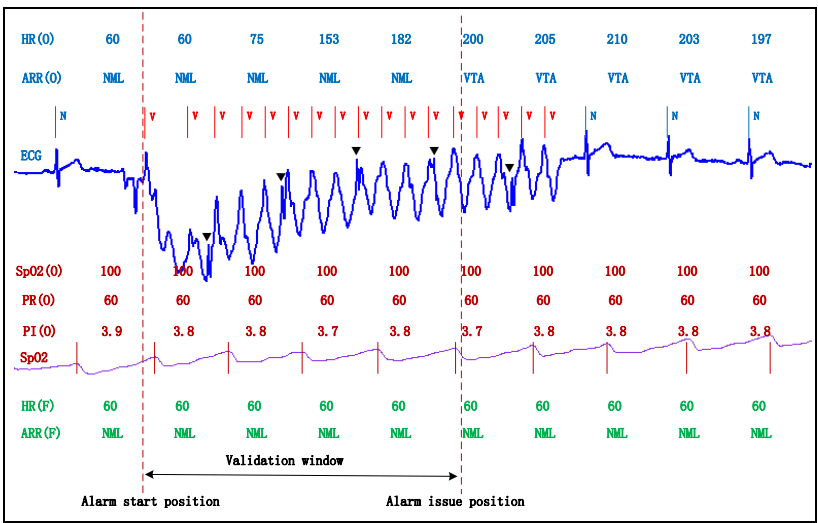

Figure 4. An example of false VTACH alarm rejection. False PVCs due to motion artifact caused a VTACH alarm. It is hard to discern if this is true or false based only on the ECG lead available. Using the SpO2 signal, spikes indicated by black triangles can be identified as actual QRSs on the ECG signal showing that the original PVC detections were unreliable and the VTACH alarm needs to be suppressed.

(3) Ventricular tachycardia (VTACH)

If the correspondence between beats classed as premature ventricular complexes (PVC) on the ECG and pulses on the $\mathrm{SpO} 2$ is unstable, abnormal $\mathrm{SpO} 2$ pulses are not present, the median $\mathrm{SpO} 2$ value is more than $90 \%$, the median PI values is greater than 1.0, $80 \%$ of the pulses indicate good quality, and the pulse rhythm is regular within the validation window, the VTACH alarm is rejected; otherwise, the alarm is retained. Figure 4 shows an example of false VTACH alarm rejection.

(4) PVC related alarms

If the SQI level is greater than 1 for over $50 \%$ of QRS complexes, spurious PVC beats have been excluded after comparison with corresponding $\mathrm{SpO} 2$ pulses, and the pulse rhythm is regular within the analysis window, the PVC related alarm is rejected; otherwise, it is retained.

(5) Missed beats

If a corresponding $\mathrm{SpO} 2$ pulse with consistent shape and interval as well as preceding pulses with good SQI exist, and PI is more than 0.7 or $\mathrm{SpO} 2$ is more than $90 \%$ within the missed beat window, the missed beat alarm is rejected; otherwise, it is retained.

(6) Rhythm related alarms

Alarms such as atrial fibrillation or irregular rhythm are associated with normal beats with irregular RR intervals. If the corresponding $\mathrm{SpO} 2$ pulse rhythm is regular, and less than $40 \%$ of the difference in pulse intervals between two consecutive pulses are greater than $80 \mathrm{~ms}$ within the alarm analysis window, the rhythm alarm is rejected; otherwise the alarm is retained.

(7) HR related alarms

When fusion indicates replacing $H R$ value by the reliable $P R$ value, HR related alarms are reappraised using the reliable PR value and retained or suppressed as appropriate.

\section{Results}

\subsection{Database Description}

Details regarding the databases used in the study are to be found in Table1. DB1 DB4 are collections of continuous ECG and $\mathrm{SpO} 2$ signals acquired using Mindray patient monitors from the CCU, NICU and PICU of 3 hospitals.

Table 1. Summary of training and test databases.

\begin{tabular}{lcccc}
\hline Dataset & DB1 & DB2 & DB3 & DB4 \\
\hline Purpose & Training & Test & Test & Test \\
Records & 465 & 1230 & 210 & 468 \\
Hours & 64 & 228 & 482 & 168 \\
$\begin{array}{l}\text { Patients } \\
\text { Evaluation }\end{array}$ & ARR & A & P/N & A \\
$\begin{array}{l}\text { Signal } \\
\text { quality }\end{array}$ & Noisy & Mixed & Mixed & Mixed \\
\hline
\end{tabular}

Note: A: Adult, P: Pediatric, N: Neonate

\subsection{Evaluation Methods}

The performance of the fusion algorithm was evaluated 
by comparing its results to those from the individual signals, ECG and SpO2. For $\mathrm{HR}$ and $\mathrm{PR}$, if the difference compared to the fusion number exceeded $20 \%$ of the lower number, the two were considered unmatched and a manual review was performed to determine which one was incorrect. For ARR alarms, a list of matched (original ECG alarm retained) and unmatched (ECG alarm suppressed) were generated and manually verified for appropriateness.

\subsection{HR/PR performance}

The reduction in the number of incorrect $\mathrm{HR} / \mathrm{PR}$ calculations for DB4 is shown in Table 2. The results indicate that the number of segments with incorrect $\mathrm{HR} / \mathrm{PR}$ is reduced significantly.

Table 2. Improvement in HR/PR accuracy.

\begin{tabular}{lcccc}
\hline \multicolumn{1}{c}{ Database } & \multicolumn{3}{c}{ DB4 } \\
\hline & \multicolumn{2}{c}{ HR } & \multicolumn{2}{c}{ PR } \\
Pre or Post Fusion & Pre & Post & Pre & Post \\
Incorrect Segments & 294 & 26 & 110 & 16 \\
Reduction ratio & \multicolumn{2}{c}{$91 \%$} & \multicolumn{2}{c}{$85 \%$} \\
\hline
\end{tabular}

\subsection{ARR alarm performance}

Tables 3 and 4 summarize the results for the suppression of false lethal (ASY, VFIB, VTACH) and other arrhythmia alarms respectively. No life-threatening true arrhythmia alarm was mistakenly rejected. The reduction in true alarms for other ARR calls was manually checked and considered acceptable given the level of reduction in false alarms.

Table 3. Reduction in false Lethal ARR alarms.

\begin{tabular}{lcccccc}
\hline Database & DB1 & \multicolumn{3}{c}{ DB2 } & \multicolumn{3}{c}{ DB3 } \\
\hline Pre or Post & Pre & Post & Pre & Post & Pre & Post \\
Fusion & 24 & 24 & 62 & 62 & 3 & 3 \\
True ARR & 24 & 68 & 209 & 64 & 52 & 8 \\
False ARR & 168 & $68 \%$ & $69 \%$ & \multicolumn{2}{c}{$85 \%$} \\
FA SR & \multicolumn{2}{c}{$60 \%$} & \multicolumn{6}{c}{6} \\
\hline
\end{tabular}

Table 4. Reduction in false calls for other ARR alarms.

\begin{tabular}{lcclccc}
\hline Database & DB1 & \multicolumn{3}{l}{ DB2 } & \multicolumn{3}{c}{ DB3 } \\
\hline Pre or Post & Pre & Post & Pre & Post & Pre & Post \\
Fusion & & & & & & \\
True ARR & 684 & 667 & 5925 & 5866 & 68 & 68 \\
False ARR & 956 & 458 & 1833 & 953 & 253 & 114 \\
FA SR & \multicolumn{2}{c}{$52 \%$} & \multicolumn{2}{c}{$48 \%$} & \multicolumn{2}{c}{$55 \%$} \\
\hline
\end{tabular}

\section{Discussion and conclusions}

In the dataset used, the ECG signal had more noise and artifact (muscle noise, bad electrodes etc.) than the $\mathrm{SpO} 2$ signal so HR values were supplanted by PR in a majority of cases. There was a larger reduction in false positive alarms corresponding to lethal rhythms than other rhythms which is to be expected. True lethal arrhythmias are typically associated with discernable changes in the $\mathrm{SpO} 2$ signal, so it is easier to separate true and false ECG based calls. If the $\mathrm{SpO} 2$ signal quality is good and hemodynamic parameters indicate robust cardiovascular circulation, rejection of false ECG calls will be reliable. Short runs of VTACH do not always cause waveform changes on the $\mathrm{SpO} 2$ signal. So, careful matching of PVCs and $\mathrm{SpO} 2$ pulses coupled with morphological analysis of PVCs is necessary to avoid suppressing true alarms. Similarly, for other PVC related alarms, confirmation using the $\mathrm{SpO} 2$ signal was carried out only when the ECG was noisy since there is no clear relation between PVC beats and corresponding pulses.

The fusion of information from ECG and $\mathrm{SpO} 2$ signals resulted in reduced false arrhythmia and $\mathrm{HR}$ alarms and increased accuracy in HR and PR in the dataset used. Since the fusion analysis used is a real-time dynamic process, it is well suited for multi-parameter patient monitors.

\section{References}

[1] ECRI Institute. Top 10 Health Technology Hazards for 2014. Health Devices 2014; 42: Issue 11.

[2] Zehui Sun et al. Reducing ECG alarm fatigue based on SQI analysis. Computing in Cardiology 2014;345-348.

[3] Jianwei Su et al. A four-lead real time arrhythmia analysis algorithm. Computing in Cardiology 2017; 1-4.

[4] Gari D Clifford et al. False alarm reduction in critical care. Physiol. Meas. 2016;37:E5-E22.

[5] Wei Zong et al. A practical algorithm to reduce false critical ECG alarms using arterial blood pressure and/or photoplethysmogram waveforms. Physiol. Meas. 2016;37:1355-1369.

[6] Qiao Li et al. Signal quality and data fusion for false alarm reduction in the intensive care unit. $\mathrm{J}$ of Electrocardiol. 2012;45;596-603.

[7] Vessela Krasteva et al. Real-time arrhythmia detection with supplementary ECG quality and pulse wave monitoring for the reduction of false alarms in ICUs. Physiol. Meas. 2016; 377:1273-1279.

[8] Zhang Xu et al. Method for measuring blood oxygen content under low perfusion. US8275434B2, 2012.

Address for correspondence.

Jianwei $\mathrm{Su}$

Mindray Building

Keji 12th Road South, High-Tech Industrial Park

Nanshan, Shenzhen, China

sujianwei@mindray.com 